\title{
ON A GENERALIZED FORM OF PLATEAU'S PROBLEM
}

\author{
BY \\ R. COURANT
}

1. Introduction. In the variational problem of Plateau and in its generalization by Douglas, minimal surfaces $S$ of least area are sought whose boundary $\Gamma$ shall consist of monotonically described curves in euclidean space with coordinates $x_{1}, x_{2}, x_{3}\left({ }^{1}\right)$. These surfaces are represented parametrically by position vectors $\mathfrak{x}(u, v)$ in a parameter domain $B$ of the $u, v$-plane with the boundary $C$, in such a way that $\mathfrak{r}$ is continuous in $B+C$ and maps $C$ monotonically on $\Gamma$. For the formulation of the problem and for the usual existence proof the monotonic character of the mapping of $C$ on $\Gamma$ is essential $\left({ }^{2}\right)$ (and implies as a consequence, incidentally, that $C$ and $\Gamma$ correspond to each other in a one-to-one way). However, from a geometrical viewpoint the restriction imposed on the parametric representation of the boundary is not natural. Therefore we replace the Plateau problem, here called Problem I, by another problem differing from the former by considerably weaker boundary conditions which do not stipulate continuity and monotonicity of the boundary mapping. This new "Problem II," to be formulated presently, is solved by the method developed in connection with "free boundaries" $\left({ }^{3}\right)$. It is the purpose of the present note to prove that the solutions of the Plateau Problem I also solve the new Problem II ; in other words, our widening of the range of competition does not affect the value of the minimum area. That this result is not obvious appears from the example at the end of this paper. We confine our proof to the case of a simply-connected surface $S$ whose parameter-do-

Presented to the Society, April 27, 1940; received by the editors May 22, 1940.

(1) The restriction to three dimensions is not essential; the reasoning applies equally well in $n$ dimensions.

(2) References-

[1] J. Douglas, Minimal surfaces of higher topological structure, Annals of Mathematics, (2), vol. 40 (1939), p. 205 ff.

[2] R. Courant, The existence of minimal surfaces of given topological structure under prescribed boundary conditions, Acta Mathematica, vol. 72 (1940), p. $51 \mathrm{ff}$.

[3] R. Courant, The existence of a minimal surface of least area bounded by prescribed Jordan arcs and prescribed surfaces, Proceedings of the National Academy of Sciences, vol. 24 (1938), pp. 97-101.

[4] R. Courant and N. Davids, Minimal surfaces spanning closed manifolds, Proceedings of the National Academy of Sciences, vol. 26 (1940), pp. 194-199.

[5] R. Courant, Plateau's problem and Dirichlet's principle, Annals of Mathematics, (2), vol. 38 (1937), p. $679 \mathrm{ff}$.

[6] R. Courant, Conformal mapping of multiply connected domains, Duke Mathematical Journal, vol. 5 (1939), p. $814 \mathrm{ff}$.

(3) See [2], [3], [4]. 
main $B$ is the unit circle. The generalization to topologically higher cases does not present any new difficulty.

2. Problem II. Generalized minimizing sequences. In the unit circle $B$ with the circumference $C$ we consider "admissible vectors" $\mathfrak{r}(u, v)$, or in polar coordinates $\mathfrak{x}(r, \theta)$, defined by the conditions: $\mathfrak{x}$ is continuous and has piecewise-continuous $\left({ }^{4}\right)$ first derivatives in $B$; the boundary of $\mathfrak{x}$ is on the prescribed Jordan curve $\Gamma$ in the $x$-space-this means that all limit points of $\mathfrak{x}(u, v)$ are on $\Gamma$ as $(u, v)$ tends to $C$; finally, instead of a monotonic mapping of $C$ on $\Gamma$, we require that the curve $\mathfrak{x}(r, \theta)$ for fixed $r$ shall be continuously deformable in to the simply described curve $\Gamma$ in such a way that for $r$ sufficiently near to 1 this deformation can be effected within an arbitrarily small neighborhood of $\Gamma$. This topological condition is equivalent to the condition that, if $H$ is any fixed simple polygon which is simply linked with $\Gamma$, the curve $\mathfrak{x}(r, \theta)$ shall, for $r$ sufficiently near to 1 , be simply linked with $H\left({ }^{5}\right)$.

Problem II requires constructing an admissible vector for which the Dirichlet in tegral

$$
D(\mathfrak{x})=\frac{1}{2} \iint_{B}\left(\mathfrak{x}_{u}^{2}+\mathfrak{x}_{v}^{2}\right) d u d v=\frac{1}{2} \int_{0}^{2 \pi} \int_{0}^{1}\left(\mathfrak{x}_{r}^{2}+\frac{1}{r^{2}} \mathfrak{x}_{\theta}^{2}\right) r d r d \theta
$$

attains a minimum value $d$. The existence of admissible vectors with finite $D(\mathfrak{x})$ is assumed, and certainly assured if $\Gamma$ is rectifiable $\left(^{6}\right)$.

Problem I differs from Problem II by the restricting assumption that $\mathfrak{x}$ is continuous in $B+C$ and maps $C$ monotonically on $\Gamma$. If $k$ is the minimum value of $D(\mathfrak{x})$ in Problem I we certainly have $k \geqq d$, since vectors admissible in I are admissible in II. Our goal is to prove $d=k$.

In [2] and [4] it is shown that Problem II is solved by a minimal surface $S$. This solution is obtained as the limit of a sequence of vectors $\mathfrak{x}_{n}$ whose members satisfy the conditions of admissibility except that the boundary of $\mathfrak{x}_{n}$ need not be exactly on $\Gamma$ but may be on a manifold $\Gamma_{n}$ which tends to $\Gamma$ as $n$ increases, such that the maximum distance of points of $\Gamma_{n}$ to $\Gamma$ tends to zero. Sequences of this type are called "admissible sequences." The smallest lower limit of $D\left(\mathfrak{x}_{n}\right)$ for all such sequences is, as seen in [4], the same value $d$ as for minimizing sequences of strictly admissible vectors. An admissible sequence $\mathfrak{x}_{n}$ for which $D\left(\mathfrak{x}_{n}\right) \rightarrow d$ is called a generalized minimizing sequence.

3 . Proof of the main theorem. For the proof we shall apply a general deformation method $\left({ }^{7}\right)$ and a theorem on conformal mapping $\left({ }^{8}\right)$. To avoid non-

(4) This means that in any closed subdomain the continuity may be interrupted at a finite number of points or arcs with continuously turning tangent.

(5) As to the essential character of such linking conditions, compare [2], [4].

(6) See, e.g., [2], p. 54, Footnote 2.

( 7 See [5], pp. 695, 707, and [6], p. 820 .

(8) Also applied in [2] and [5]. 
essential technical complications we make the assumption that $\Gamma$ is piecewise smooth, i.e., consists of a finite number of arcs having tangents continuous including the end-points.

In [2] and [4] it is shown that every generalized minimizing sequence $\mathfrak{x}_{n}$ can be replaced by such a sequence of harmonic vectors, which has a subsequence converging to a solution of II. The parameters $u, v$ of each vector may be so chosen that the origin always corresponds to a point on the polygon $H$. In [2] and in the previous literature the following theorem on Problem I is established: if $\Gamma_{n}$ is a sequence of simple polygons converging to $\Gamma$ in the Fréchet sense, and if $z_{n}$ is admissible in Problem I for $\Gamma_{n}$, then the solution $z$ of $\mathrm{I}$ for $\Gamma$ satisfies the inequality

$$
D(z) \leqq \lim \inf D\left(z_{n}\right) .
$$

The goal of this paper is therefore attained if we construct a generalized minimizing sequence for II consisting of such vectors $z_{n}(u, v)$ which map $C$ continuously and monotonically on $\Gamma_{n}$, and hence are admissible in the variational Problem I for the contour $\Gamma_{n}$.

The construction of $z_{n}$ will proceed in two steps. If the harmonic vector $\mathfrak{x}(u, v)$, or in polar coordinates $\mathfrak{x}(r, \theta)$, is a solution of Problem II with $D(\mathfrak{x})=d$ and if $r_{n}<1$ is a sequence of radii tending to 1 , then $\mathfrak{x}_{n}(r, \theta)=\mathfrak{r}\left(r / r_{n}, \theta\right)$ is certainly a generalized minimizing sequence; the boundary $\mathfrak{x}_{n}(1, \theta)$ is an analytic curve $\Lambda_{n}$ which, for given small $\epsilon=\epsilon_{n}$ and sufficiently large $r_{n}$, will be everywhere nearer to $\Gamma$ then $\epsilon / 2$.

We inscribe in $\Gamma$ a polygon $\Gamma_{n}$ whose edges have a length between $\epsilon^{1 / 2}$ and $2 \epsilon^{1 / 2}$. By choosing the vertices of $\Gamma_{n}$ on $\Gamma$ and by choosing a suitably large unit length in the $x$-space we are sure that for sufficiently small $\epsilon$ the distance from every point of $\Gamma_{n}$ to $\Gamma$ is less than $\epsilon / 2$, therefore to $\Lambda_{n}$ less than $\epsilon$. (Here the piecewise smoothness of $\Gamma$ is used to ensure that the distance between $\Gamma$ and $\Gamma_{n}$ is small of a higher order than the edges of $\Gamma_{n}$.) For $\epsilon=\epsilon_{n}$ we choose a sequence tending to zero, so that $\Gamma_{n}$ tends to $\Gamma$ in the Fréchet sense; $r_{n}, \mathfrak{x}_{n}, \Lambda_{n}$ are defined accordingly.

Now the first step of the construction consists in replacing the sequence $\mathfrak{x}_{n}$ by another generalized minimizing sequence $\mathfrak{y}_{n}(u, v)=\mathfrak{y}_{n}(r, \theta)$ such that $\mathfrak{y}_{n}$ is continuous in $B+C$ and maps $C$ on the polygon $\Gamma_{n}$ instead of on $\Lambda_{n} \cdot \mathfrak{y}_{n}(1, \theta)$ is piecewise analytic in $\theta$ but not necessarily monotonic. The second step consists in showing that the sequence $\mathfrak{y}_{n}$ may be replaced by another generalized minimizing sequence $z_{n}$ with the same properties as $\mathfrak{y}_{n}$, but mapping $C$ monotonically on $\Gamma_{n}$. Our main theorem will then be proved.

We start with the second step, assuming that the sequence $\mathfrak{y}_{n}$ has already been constructed. If $\mathfrak{y}=\mathfrak{y}_{n}(r, \theta)$ has piecewise analytic boundary values $\mathfrak{y}(1, \theta)$ which describe the polygon $\Gamma_{n}$ not monotonically, there are but a finite number of "stationary" $\theta$-intervals along which $\mathfrak{y}$ is a constant and a finite number of "folds," i.e., parts of $\Gamma_{n}$ which are described more than 
once and in different directions. We eliminate the stationary intervals and folds successively by conformal mapping. If $A_{0} A_{1}: \theta_{0} \leqq \theta \leqq \theta_{1}$, is a stationary interval $s$ on $C$, we map the circle $B$ conformally on another unit circle $B^{\prime}$ with a slit $s^{\prime}$ so that the slit $s$ corresponds to the arc $s$. This mapping transforms $y$ in $B$ into $\mathfrak{y}^{\prime}$ in $B^{\prime}-s^{\prime}$ and, because of the invariance of the Dirichlet integral, we have

$$
D_{B^{\prime}-\mathbf{s}^{\prime}}\left(\mathfrak{y}^{\prime}\right)=D_{B}(\mathfrak{y}) \text {. }
$$

But, since $\mathfrak{y}^{\prime}$ is constant on $s$ it is continuous in $B^{\prime}$ including its boundary. Denoting $B^{\prime}$ and $\mathfrak{y}^{\prime}$ again by $B$ and $\mathfrak{y}$, we have replaced $\mathfrak{y}=\mathfrak{y}_{n}$ by another vector with the same properties and at the same time reduced the number of stationary intervals by one. Repeating this process we eliminate in $\mathfrak{y}$ all stationary intervals.

The folds are treated in a similar way. Suppose the interval s: $A_{0} P A_{1}$ on $C$ corresponds to a fold such that the arcs $A_{0} P$ and $P A_{1}$ are mapped analytically on the same portion of an edge of $\Gamma_{n}$, each arc monotonically but the two arcs with different sense of description. Then those points on $A_{0} P$ and $P A_{1}$ which have the same image point on $\Gamma_{n}$ correspond to each other by an analytic transformation of $\theta$. Hence, according to a procedure used in previous publications $\left(^{9}\right)$ (sewing theorem) we can identify such corresponding points by conformal mapping. Namely, we can $\operatorname{map} B$ on a unit circle $B^{\prime}$ with an analytic slit $s^{\prime}$ so that the interiors of the arcs $A_{0} P$ and $P A_{1}$ correspond to the two edges of $s^{\prime}$ and that corresponding points on $s$ go into opposite points on the two edges of $s^{\prime}$. The end-points $A_{0}, A_{1}, P$ are transformed into points or possibly into analytic slits. Again, $\mathfrak{y}$ is transformed into $\mathfrak{y}^{\prime}$ in $B^{\prime}-s^{\prime}$ and the reasoning proceeds as above, since $\mathfrak{y}^{\prime}$ remains continuous in $B^{\prime}$ after erasing $s^{\prime}$. In this way, by a finite number of steps, all the folds are eliminated without any change of the Dirichlet integral. We therefore obtain, starting from $\mathfrak{y}_{n}$ a new generalized minimizing sequence $z_{n}$ as stipulated.

The remaining step, i.e., the construction of $\mathfrak{y}_{n}$ from $\mathfrak{x}_{n}$, is done by a general variational method $\left({ }^{10}\right)$. We consider the influence of certain deformations of the whole $x$-space on the variational integral. In our case these deformations are to affect only the neighborhood of $\Gamma$. They deform the analytic curve $\Lambda_{n}$ given by $\mathfrak{x}\left(r_{n}, \theta\right)$ into the polygon $\Gamma_{n}$. Considering those points in the $x$-space which are images under $\mathfrak{x}_{n}(u, v)$ of points in $B$, we transform $\mathfrak{x}_{n}(u, v)$ into a vector $\mathfrak{y}_{n}(u, v)$, and we shall see to it that $\mathfrak{y}_{n}$ is again a generalized minimizing sequence. Our transformations will be described as composed of a finite number of "pinching processes":

(a) First, " $\delta$-pinching around a point $P$ " is defined as follows: assuming $P$ to be the origin $x_{1}=x_{2}=x_{3}=0$, we define, for a given small $\delta$ and $t=t(\delta)>1$,

(') See [2], p. $57 \mathrm{ff}$.

(10) See [2], p. 81. 
the vector

where $r^{2}=\sum x_{i}^{2}$ and

$$
y_{i}=p(r) x_{i}
$$

$i=1,2,3$,

$$
\begin{gathered}
p=1 \text { for } r>t \delta ; \quad p=0 \text { for } r<\delta ; \\
p=\sigma \log \frac{r}{\delta} \text { for } \delta<r<t \delta
\end{gathered}
$$

with $\sigma=1 / \log t$. In other words, the deformation pinches the $\delta$-sphere around $P$ into $P$, leaves points outside of the larger sphere with the radius $t \delta$ unchanged, and moves every point on a straight line towards $P$. Substituting for $x_{i}$ functions of $u$ and $v$ we obtain $y_{i}$ as functions of $u$ and $v$ and thus a vector $\mathfrak{y}(u, v)$.

To compare the Dirichlet integral of $\mathfrak{y}$ with that of $\mathfrak{x}$ we consider in $B$ the subset $B^{*}$ where $p<1$, i.e., those points $u, v$ whose images under $\mathfrak{x}(u, v)$ are nearer to $P$ than $t \delta$. Let $D^{*}=D_{B^{*}}$ denote the Dirichlet integral over $B^{*}$. We then have, since $|\mathfrak{x}|=r,\left|x^{i}\right| \leqq r,|p| \leqq 1\left({ }^{11}\right)$,

$$
\begin{aligned}
D^{*}(\mathfrak{y}) \leqq & \frac{1}{2} \iint_{B^{*}} \sum_{i=1}^{3}\left[\left\{\left|p x_{u}^{i}\right|+\left|\sigma x^{i}\right| \frac{1}{r^{2}}\left|\mathfrak{x x}_{u}\right|\right\}^{2}\right. \\
& \left.+\left\{\left|p x_{v}^{i}\right|+\left|\sigma x^{i}\right| \frac{1}{r^{2}}\left|\mathfrak{x}_{v}\right|\right\}^{2}\right] d u d v \\
\leqq & \frac{1}{2} \iint \sum\left[\left\{\left|x_{u}^{i}\right|+\sigma\left|\mathfrak{x}_{u}\right|\right\}^{2}+\left\{\left|x_{v}^{i}\right|+\sigma\left|\mathfrak{x}_{v}\right|\right\}^{2}\right] d u d v \\
\leqq & \frac{1}{2} \iint\left(\mathfrak{x}_{u}^{2}+\mathfrak{x}_{v}^{2}\right)\left(1+3 \sigma^{2}+4 \sigma\right) d u d v
\end{aligned}
$$

hence

(1) $D^{*}(\mathfrak{y}) \leqq \frac{1}{2} \iint_{B^{*}}(1+3 \sigma)^{2}\left(\mathfrak{x}_{u}^{2}+\mathfrak{x}_{v}^{2}\right) d u d v=(1+3 \sigma)^{2} D^{*}(\mathfrak{x})$

which shows that for small $\sigma$ the influence of the transformation on the Dirichlet integral is small. We may write

$$
D(\mathfrak{y}) \leqq \frac{1}{2} \iint_{B}(1+\eta)^{2}\left(\mathfrak{x}_{u}^{2}+\mathfrak{x}_{v}^{2}\right) d u d v
$$

where $\eta=0$ outside $B^{*}$ and

$$
|\eta| \leqq 3 \sigma(\delta)
$$

elsewhere.

(11) The coordinate indices are written as superscripts here and immediately below. 
(b) Secondly, we introduce " $\delta$-pinching around a straight segment," for which, without restriction of generality, we may choose

$$
s: \quad x_{2}=x_{3}=0 ; \quad\left|x_{1}\right|<a+\alpha ;
$$

$t=t(\delta)$ and $\sigma$ are defined as above and we assume

$$
|a|>t \delta, \quad|\alpha|>t \delta .
$$

We define the transformation for $\left|x_{1}\right|<a$ by

$$
y_{1}=x_{1}, \quad y_{2}=p(\rho) x_{2}, \quad y_{3}=p(\rho) x_{3},
$$

with $\rho^{2}=x_{2}^{2}+x_{3}^{2}$ and $p(\rho)$ as above. For $x_{1}>a$ and $x_{2}<-a$ the transformation is defined as $\delta$-pinching around the point $x_{1}=a$ and $x_{1}=-a$ on the segment $s$, respectively. By this pinching an essentially cylindrical bar around $s$ with two half-spherical ends is contracted into $s$.

Again a simple appraisal shows that, for the subdomain $B^{*}$ of $B$ to which vectors $\mathfrak{x}$ affected by our pinching correspond, we have the same inequality (1) or (2) as in the pinching around a point.

(c) By carrying out successively $N$ pinchings around different points and lines, possibly with different values of $\delta=\delta_{i}$, we obtain from $\mathfrak{x}$ a vector $\mathfrak{y}$ with

$$
D(\mathfrak{y}) \leqq \frac{1}{2} \iint_{B}\left(1+\eta_{1}\right)^{2} \cdots\left(1+\eta_{N}\right)^{2}\left(\mathfrak{x}_{u}^{2}+\mathfrak{x}_{i}^{2}\right) d u d v
$$

where the $\eta_{i}$ are defined as above. If no more than two quantities $\eta_{i}$ differ from zero at the same point, we have

$$
D(\mathfrak{y}) \leqq(1+3 \sigma)^{4} D(\mathfrak{x})
$$

where $\sigma$ now denotes the largest value $1 / \log t_{\nu}$ employed in our pinchings.

(d) In applying the preceding results we consider, as above, a sequence of small values $\epsilon=\epsilon_{n}$ tending to zero, and corresponding polygons $\Gamma_{n}$ and analytic curves $\Lambda_{n}$ representing the boundary of $\mathfrak{x}_{n}$. We transform, for sufficiently large $n$ and small $\epsilon=\epsilon_{n}, \mathfrak{x}_{n}$ in to another vector $\mathfrak{y}_{n}$ as follows: with

$$
\tau=\epsilon^{1 / 8}, \quad \delta=\tau^{6}, \quad t=\tau^{-1}, \quad \sigma=\frac{8}{\log \epsilon^{-1}},
$$

we perform a $\delta$-pinching around each vertex of $\Gamma_{n}$. For small values of $\epsilon$ these deformations do not interfere with each other and transform $\Gamma_{n}$ into itself, since the edges of $\Gamma_{n}$ are all longer than $\tau^{4}=\epsilon^{1 / 2}$ and the angles between adjacent edges remain above a fixed positive bound. We then subject each edge of $\Gamma_{n}$, except the ends, already pinched into the vertices, to a $\delta$-pinching as under (b). In this deformation we choose as $s$ a symmetric part of the edge by cutting off from each end the length $\frac{1}{2} \tau^{6}$ so that, if $l \geqq \tau^{4}$ is the length of the edge, we have 


$$
2 a=l-\tau^{6}, \quad \delta=\tau^{8}, \quad t=\tau^{-1}, \quad \delta t=\tau^{-7} .
$$

Here the dimensions are again so balanced that for sufficiently small $\epsilon$ no interference with another edge occurs. Moreover it is obvious that the succession of all our pinchings results in a piecewise analytic deformation of $\mathfrak{x}_{n}$ in to a vector $\mathfrak{y}_{n}$ such that the analytic curve $\Lambda_{n}$ is transformed into $\Gamma_{n}$ and that the condition for the inequality (1) is satisfied for $\mathfrak{x}=\mathfrak{x}_{n}, \mathfrak{y}=\mathfrak{y}_{n}, \sigma=\sigma_{n}$ $=8 / \log \epsilon_{n}^{-1}$. Hence the $\mathfrak{y}$ are again a generalized minimizing sequence. The proof of our main theorem is thereby completed.

4. Remarks. While every solution of I is proved to be a solution of II, the converse is not necessarily true. The following example demonstrates that

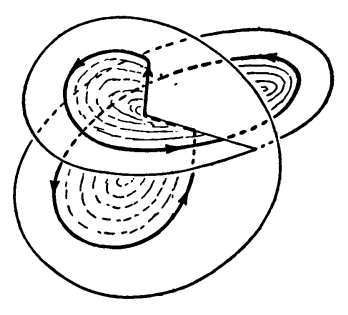

FIG. 1

for certain prescribed Jordan contours $\Gamma$ the absolute minimum in the generalized problem can be attained by a vector $\mathfrak{x}(u, v)$ which maps the circle $C$ in a continuous but non-monotonic way on $\Gamma$, such that on a part of $\Gamma$ a fold is formed.

We consider a simply-connected minimal surface $S$ with a branch point similar to that of the Riemann surface of $w^{1 / 2}$ and furnishing the absolute minimum for a Plateau Problem I $\left({ }^{12}\right)$. Any continuous, non-self-intersecting curve $\Gamma$ on $S$ obviously bounds a simply connected minimal surface $T$ on $S$

(12) Apparently no instance of a minimal surface with branchpoint has been pointed out explicitly in the literature. However, the following procedure provides infinitely many examples: We start with a triangle $O A B$ where $O A$ and $O B$ are straight segments of equal length forming an angle of 120 degrees and $A B$ an arc (e.g., circular or polygonal) not in a plane with the points $O, A, B$. This triangle bounds a minimal surface $M^{\prime}$ of least area. Now, by reflecting $M^{\prime}$ successively on its straight edges issuing from $O$ we obtain after 5 reflections a minimal surface $M$ of the desired type with $O$ as branchpoint and with three straight lines of self-intersection at angles of 120 degrees. That any simply connected portion $S$ of $M$ including the branchpoint $O$ and having a piecewise smooth boundary curve furnishes an absolute minimum for the area, is inferred, for sufficiently flat triangles $O A B$ from the classical theory. Our figure shows only the one line of self-intersection which is used for the construction.

Dr. C. L. Siegel in Princeton called the author's attention to a paper by B. Stessmann, Periodische Minimalflächen, Mathematische Zeitschrift, vol. 38 (1934), pp. 417-442, in which the Example 4 leads to a surface of the type described above. According to a personal communication, Professor Rado, using the principle of reflection, likewise has constructed an example of a simply connected minimal surface with branchpoint, bounded by a knotted clover-leaf curve. (This footnote was added January 15, 1941.) 
solving Problem I for the boundary F. Figure 1 represents such a surface $S$ topologically but with some metric exaggeration; for the sake of clarity, the curve $\Gamma$ is indicated by a heavy line. The line $\Gamma$ goes through the branch point and follows along a segment of a line of self-intersection $c$ of $S$. $T$ is represented separately in Figure 2. The portion $T$ on $S$ to the right of $\Gamma$ has the

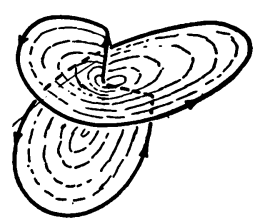

FIG. 2

minimum area $k$ for Problem I. On the other hand we may, instead, cut the surface $T$ along the line of intersection $c$ as far as $c$ coincides with $\Gamma$. $T$ then becomes another simply connected domain $T^{\prime}$ with the same area and the same boundary $\Gamma$, except that the part of $\Gamma$ along this cut now is described once in one direction and twice in the other, as indicated in the Figure 3. $T$ is therefore a solution of II which is not a solution of $\mathrm{I}$.

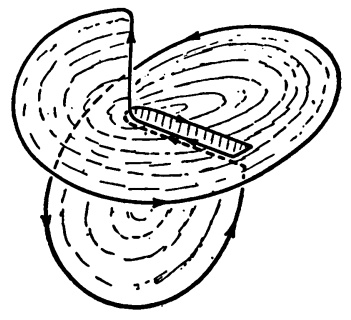

FIG. 3

NEW YORK UNIVERSITY

NEw YoRK, N. Y. 